FORMATION Formation emploi

Revue française de sciences sociales

154 | Avril-Juin

Le handicap face à la formation et au travail : vers une employabilité inclusive?

\title{
Présentation de l'ouvrage de Laure Minassian, L'enseignement professionnel entre promotion et relégation. Une approche sociologique
}

Séverine Depoilly

\section{OpenEdition}

\section{Journals}

Édition électronique

URL : https://journals.openedition.org/formationemploi/9399

DOI : 10.4000/formationemploi.9399

ISSN : 2107-0946

Éditeur

La Documentation française

Édition imprimée

Date de publication : 21 septembre 2021

Pagination : 223-226

ISSN : 0759-6340

Référence électronique

Séverine Depoilly, «Présentation de l'ouvrage de Laure Minassian, L'enseignement professionnel entre promotion et relégation. Une approche sociologique», Formation emploi [En ligne], 154 | Avril-Juin, mis en ligne le 21 septembre 2021, consulté le 06 janvier 2023. URL : http://journals.openedition.org/ formationemploi/9399; DOI : https://doi.org/10.4000/formationemploi.9399 


\title{
Note de Lecture
}

\section{Présentation de l'ouvrage de Laure Minassian, L'enseignement professionnel entre promotion et relégation. Une approche sociologique}

\begin{abstract}
Par Séverine Depoilly Maîtresse de conférences en sociologie. Laboratoire du Groupe de Recherches Sociologiques sur les Sociétés Contemporaines (Gresco), Université de Poitiers
\end{abstract}

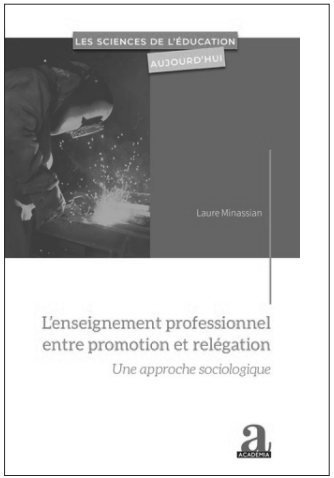
grands moments, chacun de ces temps armant le lecteur de connaissances historiques, sociologiques, sur les grandes étapes de la structuration de l'enseignement professionnel, sur la spécificité de ses publics, des contenus de connaissances et de savoirs transmis.

\section{1. « Histoire et politiques éducatives de l'enseignement professionnel en France et en Europe »}

La première partie de l'ouvrage est composée de quatre chapitres. Elle propose d'abord une mise en perspective historique de l'enseignement professionnel. Le chapitre 1 s'intéresse ainsi notamment aux politiques publiques d'éducation qui ont participé à sa structuration et qui l'ont progressivement fait passer d'un ordre d'enseignement sélectif, chargé de la formation et de la qualification d'une élite ouvrière, à un segment du système éducatif plutôt situé dans le bas des hiérarchies socio-scolaires. 
Pour mieux comprendre ce mouvement, l'autrice revient tant sur les transformations des mondes économiques et du travail que sur l'ampleur du mouvement de démocratisation scolaire qui a directement impacté l'enseignement professionnel. De fait, si ce mouvement de démocratisation a servi la politique du $80 \%$ d'une classe d'âge au bac, elle a aussi déplacé les sas de sélection vers l'enseignement professionnel.

Dans les chapitres 2 et 3, Laure Minassian invite les lecteurs à faire un pas de côté grâce à la comparaison internationale. Ce déplacement permet, d'une part, de spécifier les singularités de l'enseignement professionnel français; d'autre part, de mieux contextualiser les modalités d'organisation concrète et les visées de l'enseignement professionnel selon les contextes géographiques et les traditions de formation dans lesquels il s'insère.

Dans l'ensemble des pays considérés, de l'Europe du Nord au Québec, l'enseignement professionnel apparaît bel et bien comme travaillé par un ensemble de préoccupations politiques et économiques qui visent tout à la fois la lutte contre le chômage des jeunes et l'élévation de leurs niveaux de formation et de qualification.

Cette mise en perspective permet au lecteur de disposer de points de repères sur l'organisation des systèmes d'enseignement professionnel. Elle permet aussi, dans une certaine mesure, de démythifier l'évidence de l'efficacité des systèmes d'enseignement professionnel, notamment de ceux situés Outre-Rhin.

\section{2. « La sélection par la culture scolaire, l'origine économique et sociale »}

La seconde partie de l'ouvrage comporte quatre chapitres et s'intéresse plus particulièrement aux pratiques pédagogiques à l'œuvre dans l'enseignement professionnel. Plus précisément, l'autrice souligne l'importance de considérer certaines des typicités de l'enseignement professionnel qui se situent notamment dans la porosité entre monde du travail et monde de l'école. En effet, dans l'enseignement professionnel par voie scolaire ou par apprentissage, les élèves, les apprentis, fréquentent l'école ou le centre de formation et l'entreprise. Des savoirs scolaires et professionnels circulent d'un univers à un autre.

De fait, pour mieux saisir cette singularité des situations et des contenus d'enseignementapprentissage dans l'enseignement professionnel, Laure Minassian revient d'abord (chapitre 1) sur ce qui caractérise la forme scolaire « classique » qui engage un certain rapport à l'écrit, au temps, aux espaces, au corps, aux autres, aux savoirs. Une des spécificités de l'enseignement professionnel, qui tend à le distinguer de l'enseignement général, réfère aux savoirs et à leur mode de transmission par voir-faire et ouï-dire.

Plus précisément, dans le chapitre 2, l'autrice rappelle la place qu'occupe l'écrit dans l'enseignement professionnel, qui n'est pas négligeable. L'enseignement professionnel est ainsi "mis au défi», comme l'écrit l'autrice. Il fait cohabiter une mise en forme classique 
des savoirs théoriques avec des savoirs plus expérientiels. Ces savoirs d'expérience ou de métiers doivent viser la généricité. Pourtant, ils restent souvent encastrés dans la pratique de l'ici et maintenant des situations productives, empêchant par là même le processus de secondarisation d'opérer. Comme le rappelle l'autrice, ce processus est essentiel à l'activité intellectuelle et à l'engagement des élèves dans l'activité d'apprentissage.

Pour mieux illustrer son argumentaire, Laure Minassian prend appui, dans son chapitre 3, sur des enquêtes conduites dans des classes de baccalauréat professionnel agricole dans un lycée professionnel (LP) et dans une une Maison familiale rurale (MFR). Si le LP offre une formation scolarisée, la MFR s'organise suivant les modalités de l'apprentissage. L'autrice prend plus précisément appui sur les usages de l'écrit dans une discipline spécifique, la zootechnie, qui suppose la maîtrise de savoirs théoriques et techniques. L'analyse comparative proposée des supports de cours, des manières de transmettre les savoirs par les enseignants sur ces deux terrains que sont le LP et la MFR montre que les mises en forme et en mots des savoirs scolaires peuvent varier et de fait produire ou reconduire des inégalités scolaires d'apprentissage. Le LP, désigné par l'autrice comme forme scolarotechnique, se distingue de la forme pratico-scolaire de la MFR. Dans ces deux structures de formation, les usages de l'écrit, les sollicitations cognitives des élèves qu'ils engagent ne permettent pas le même travail de secondarisation des savoirs; ce travail nécessitant la mise à distance de la pratique pour que puissent en être généralisées certaines des composantes qui deviennent alors transférables à d'autres contextes et domaines de pratiques.

Comme le note l'autrice, ces deux formes obligent à considérer que les activités cognitives sollicitées chez les élèves et les apprentis sont inégalement rentables et ne permettent pas le même accès à une culture technique possiblement émancipatrice.

\section{3. « Le devenir des anciens élèves »}

Dans la troisième et dernière partie de l'ouvrage, Laure Minassian propose d'explorer plus avant les hypothèses précédemment formulées en s'intéressant aux devenirs des élèves sortant de LP et de MFR. Elle montre ainsi que les parcours des sortants de ces formations sont loin d'être homogènes et ne sont pas, de fait, enfermés dans une indépassable expérience de la domination.

Examinant, dans une perspective quantitative (chapitres 1 et 2), puis qualitative (chapitre 3), les types de poursuite d'études dans l'enseignement supérieur, les conditions d'insertion sur le marché de l'emploi selon que les jeunes sont bacheliers ou apprentis, filles ou garçons, qu'ils ont suivi une formation industrielle ou tertiaire, ou encore selon leur catégorie sociale d'origine, l'autrice montre comment chacune de ces dimensions de l'expérience impacte les conditions plus ou moins facilitées d'accès à l'emploi.

Ainsi, les lycéens professionnels apparaissent particulièrement fragiles dans l'enseignement supérieur, notamment dans les filières de STS (sections de technicien supérieur), 
quand les apprentis semblent pouvoir connaittre une insertion professionnelle facilitée par leur expérience des environnements de travail. Les grandes perdantes semblent bel et bien être les femmes. Plus massivement scolarisées dans des filières tertiaires aux débouchés incertains, moins souvent en apprentissage que leurs homologues masculins, elles ne bénéficient pas des mêmes horizons scolaires et professionnels.

Dans un dernier chapitre (le chapitre 4), l'autrice donne chair à ses précédentes analyses en proposant de revenir plus précisément sur le parcours de quinze jeunes ruraux d'origine populaire qui ont suivi leur formation dans l'enseignement professionnel agricole. Lanalyse de ces parcours, saisis sur un empan temporel de neuf années, permet de montrer qu'il existe différentes formes de mobilité sociale et spatiale qui vont de la non-entrée dans la carrière d'exploitant à l'accomplissement d'une carrière d'exploitant ou de cadre dans de grandes coopératives agricoles.

Lautrice souligne l'importance, pour comprendre les formes d'engagement ou de désengagement dans la carrière, de l'impact de différents types de ressources pouvant être mobilisées : l'appartenance de genre, l'expérience en stage pour les apprentis, le rôle d'un capital culturel, d'un patrimoine ou encore de la certification corporative.

Au final, l'ouvrage proposé par Laure Minassian, relativement court, n’en est pas moins dense. Il offre un panorama assez complet, mais aussi original, des problématiques qui traversent l'enseignement professionnel en France. Les analyses proposées par l'autrice sur ce que l'enseignement professionnel fait aux élèves au prisme des contenus de savoirs, de leur mise en forme dans l'activité d'enseignement-apprentissage, des modalités et des conditions de leur transmission et de leur appropriation, offrent des perspectives encore trop peu explorées aujourd'hui.

Cette approche par les savoirs nous semble de fait pouvoir permettre de dépasser l'éternelle question posée à l'enseignement professionnel : permet-il de s'émanciper ou est-il un simple instrument de relégation et de domination?

Cette question, certainement trop simple, mais pourtant reprise par Laure Minassian dans le titre de son ouvrage, ne rend pas suffisamment justice aux multiples pistes que ce dernier propose d'explorer pour mieux comprendre et mesurer la complexité propre à l'enseignement professionnel.

\section{Référence de l'ouvrage}

Minassian L. (2020), L'enseignement professionnel entre promotion et relégation. Une approche sociologique, Louvain-La-Neuve, Ed. Academia-L'Harmattan, Coll. " Les sciences de l'éducation aujourd'hui ». 